\title{
Kernos
}

Revue internationale et pluridisciplinaire de religion grecque antique

9 | 1996

Varia

\section{La notion d'harmonie dans la pensée politique de Solon}

\author{
Louise-Marie L'Homme-Wéry
}

\section{OpenEdition}

Journals

\section{Édition électronique}

URL : http://journals.openedition.org/kernos/1164

DOI : 10.4000/kernos. 1164

ISSN : 2034-7871

\section{Éditeur}

Centre international d'étude de la religion grecque antique

\section{Édition imprimée}

Date de publication : 1 janvier 1996

ISSN : 0776-3824

\section{Référence électronique}

Louise-Marie L'Homme-Wéry, "La notion d'harmonie dans la pensée politique de Solon », Kernos [En ligne], 9 | 1996, mis en ligne le 21 avril 2011, consulté le 19 avril 2019. URL : http:// journals.openedition.org/kernos/1164; DOI : 10.4000/kernos.1164 
Kernos, 9 (1996), p. 145-154.

\section{La notion d'harmonie}

\section{dans la pensée politique de Solon}

Que la notion d'harmonie soit fondamentale dans la pensée politique de Solon, c'est ce dont témoigne l'emploi du vocabulaire dans ses poèmes politiques. Non pas que les occurrences des mots composés à partir de la racine ar-y soient nombreuses, mais il est significatif qu'elles y elles occupent une position-clé et qu'elles s'y présentent, d'une part, lorsqu'il définit la cité idéale, et, d'autre part, lorsqu'il rappelle la manière dont il l'a réalisée'.

Lorsqu'avant la promulgation de sa législation, Solon décrit l'Athènes de l'Eunomia, de la «bonne organisation civique $»^{2}$, il la définit d'abord comme celle de l'ordre et de l'harmonie ${ }^{3}$ :

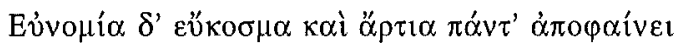

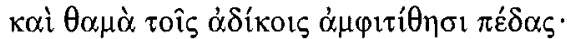

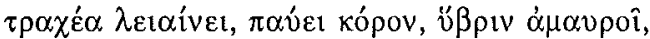

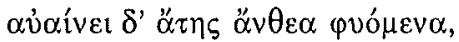

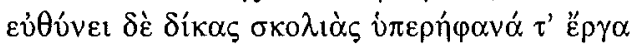

1 SOLON, fr. 3, 32 et 39 G.-P., 5, 5 G.-P. et 8, 4 G.-P. emploient l'adjectif ä $\rho \tau 1 o \zeta ;$ SOLON, fr. 30,16 G.-P. le verbe $\xi v v \alpha \rho \mu o ́ \zeta \omega$ et fr. 30, 19 G.-P. le verbe $\alpha \rho \mu o ́ \zeta \omega$ (l'édition des poèmes de Solon qui est ici utilisée est celle de B. GENTILI et C. PRATO, Poetarum Elegiaconum Testimonia et Fragmenta, I, Teubner, $1988^{2}$, p. 93-126). - E. BENVENISTE, Le vocabulatre des institutions indo-européennes, II, Paris, 1969, p. 99-105, a établi que « la racine commune à skr. rta, ir. arta, lat. ars, artus, ritus, désigne 1'“ordre" comme adaptation harmonieuse des parties d'un tout entre elles ". C. CALAME, Die Komposita mit $\alpha \rho \tau$ - im frilbgriecbiscben Epos, in $M H, 34$ (1977), p. 209-220, a montré, par une étude systématique des termes formés à partir de la racine $a r$-dans les poèmes homériques et hésiodiques, que cette disposition harmonieuse s'y rapportait aussi bien à des particularités humaines (pieds, esprit, parole) qu'à l'entente affective, et à tout ordre, quel qu'il soit (militaire, social) sans que le mot comporte une référence à l'éthique. Ainsi en $I l$, XXII, 281, l'adjectif

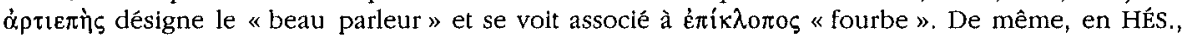

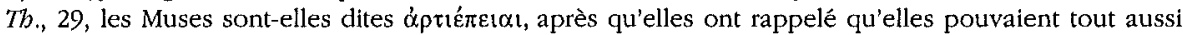
bien conter de nombreux mensonges semblables à des réalltés que, proférer, lorsqu'elles le

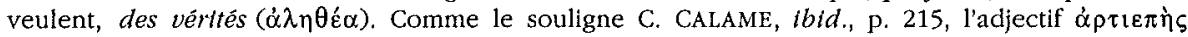
qualifie donc ici leur savoir-faire, non leur aptitude à dire toujours vrai.

2 Même si le sens du mot etmomia reste discuté, en fonction des applications diverses qui ont été données de ce concept, les mots de la racine nem-contiennent toujours, comme l'a montré E. LAROCHE, Histoire de la racine nem-en grec ancien, Paris, 1949, l'idée de répartition, de partage, ce qui autorise à traduire eunomia par bonne organisation, bon partage.

3 SOLON, fr. 3, 32 G.-P. = DÉM., 19 (Leg.), 255. 


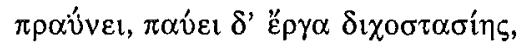

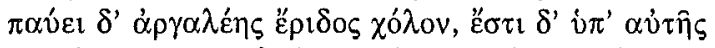

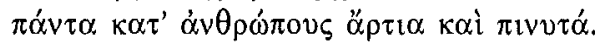

Eunomia, elle, révèle que toute chose est bien ordonnée et bien agencée. Vite, elle met des entraves aux pieds des gens injustes. Ce qui est abrupt, elle l'adoucit, elle fait cesser la convoitise, elle émousse l'orgueil, elle dessèche les fleurs naissantes de la folie. Elle redresse les jugements torves. Les actes de la superbe, elle les adoucit. Elle met fin aux œuvres de la stasis qui oppose les deux partis, elle fait cesser le fiel de la pénible discorde. Sous son règne, chez les hommes, tout est harmonieux, tout est inspiré.

L'adjectif $\alpha \rho \tau \imath \alpha$, présent au premier vers, comme au dernier vers de cette description, détermine les traits essentiels de cette Eunomia, cette bonne organisation civique, que Solon conçoit comme marquée par les valeurs corrélatives d'ordre et d'harmonie. La cité idéale est pour lui celle où un ordre harmonieux, voulu par les dieux, se trouve atteint, de telle sorte que la justice y est réalisée (plus de jugements torves), et la paix intérieure assurée (plus de stasis). Ainsi déjà, dans la Théogonie d'Hésiode, Dikè, Eirènè, Eunomia, sontelles sœurs. Filles de Zeus et de Thémis, elles sont les Heures, source de cet ordre idéal qui assure au paysan la prospérité de ses champs ${ }^{4}$.

Cependant cet ordre qu'Hésiode appelle de ses vœux, mais sans pouvoir le réaliser autrement que par la vision mythique qu'il en donne, Solon entreprend de le concrétiser à Athènes par une œuvre double dont les deux pans essentiels - libération et législation - sont eux-mêmes associés dans sa pensée à la notion d'harmonie. Libérant Athènes et les Athéniens d'une domination, dont on a montré ailleurs qu'elle était celle que les Mégariens avaient établie sur la plaine thriasienne ${ }^{5}$, il rappelle à ses concitoyens qu'il a accompli cette œuvre comme un harmonisateur qui concilie bia et $d i k e^{6}$ :

$\tau \alpha \hat{v} \tau \alpha \mu \grave{\varepsilon} v \kappa \rho \alpha ́ \tau \varepsilon 1$,

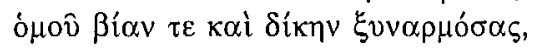

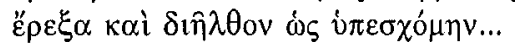

\footnotetext{
4 HÉs, Th., 900-903.
}

5 L'identité entre la Terre Noire et celle d'Éleusis a été établie par H. VAN EFFENTERRE, Solon et la terre d'Eleusis, in RIDA, 24 (1977), p. 91-129. - J'ai montré que cette libération jouait un rôle fondamental dans la politique de Solon, même si la tradition athénienne se tait sur ce point (Louise-Marie L'HOMME-WÉRY, La perspective éleusinlenne dans la politique de Solon, à paraître dans la Bibliotbèque de la Faculté de Pbilosopbie et Lettres de l'Université de Liège).

6 SOLON, fr. 30, 15-20 G.-P. $=A . P .$, XII, 4. Au vers 15, les éditeurs sont divisés entre les lectures vónov et ópov̂ ( $c f$. P.J. RHODES, A Commentary on the Aristotelian Athenaion Politela, Oxford, $1985^{2}$, p. 176). Cependant seul thesmos au pluriel est employé par Solon pour désigner ses lois (SOLON, fr. 30,17 G.-P. et 40,1 G.-P.), ce qui permet de trancher sans hésitation possible en faveur de ópov̂. 
Cela, je l'ai fait, par mon pouvoir, en harmonisant l'une à l'autre violence et justice, et j'ai été jusqu'au bout, comme je l'avais promis.

La force coercitive qu'il détient, le pouvoir militaire qu'il exerce pour réaliser cette libération se justifient donc à ses yeux par la justice qu'il estime atteindre grâce à eux. Polémarque pour libérer Eleusis, diallaktès en 594/3 pour régler les problèmes de repartage qui résultent de cette libération, et libérer les Athéniens victimes de la domination mégarienne, Solon se veut harmonisateur d'Athènes, réalisateur de la justice, même si c'est par la violence que cette justice a été atteinte ${ }^{7}$. De même, quand il promulgue les lois, le fait-il, dit-il, pour harmoniser, pour «ajuster une justice droite » pour chaque Athénien, quel qu'il soit, kakos ou agatbos ${ }^{8}$ :

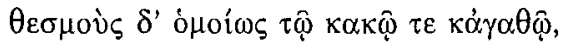

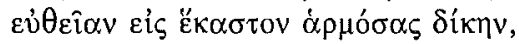
है $\gamma \rho \alpha \psi \alpha$.

Quant aux lois, c'est semblablement que, pour l'homme de rien comme pour le noble, je les ai écrites, harmonisant pour chacun une justice droite.

Ainsi la loi, thesmos, en unissant sous sa règle les deux classes opposées d'Athènes, y accomplit-elle cette justice que Solon envisage comme le bien ultime de la cité et dont il se veut le réalisateur, tant dans sa promulgation des lois que dans la libération de la Terre Noire d'Eleusis qui l'a précédée. Ces deux parties de son cuvre forment d'ailleurs à ses yeux un tout, comme il le souligne en enchaînant l'une à l'autre, dans ces trimètres iambiques où, par la symétrie du style, il souligne l'unité de l'œuvre'. Harmonisateur pour libérer Eleusis et les victimes de la domination mégarienne, il voit dans ce rétablissement de l'intégrité civique l'accomplissement de la justice; de même est-ce cette justice qu'il prétend accomplir quand il écrit les lois d'Athènes en une autre œuvre d'harmonisation qui, comme la première, se veut son œuvre exclusive : dans ces trimètres iambiques, les verbes ěp $\varepsilon \xi \alpha$, Ě $\gamma \rho \alpha \psi \alpha^{10}$, fortement soulignés par leur position en début de vers, sont des singuliers. Ainsi Solon entend-il façonner Athènes en une ouvre solitaire qui prétend transcrire dans

7 HÊs., Tr., 274-280, oppose bia, loi du monde sauvage où les animaux se dévorent entre eux et dikè, loi établie par le Cronide pour les hommes.

8 SOLON, fr. 30, 15-20 G.-P. = A.P., XII, 4 et PLUT., Sol, 15, 1..

9 Le parallélisme qui s'établit au niveau du style entre les vers 15 à 17 , et 18 à 20 de SOLON, fr. 30 G.-P. a été souligné par Nicole LORAuX, Solon el la volx de l'écrit, in M. DETIENNE (éd.), Les Savoirs de l'écriture. En Grèce Ancienne, Lille, 1989, p. 123-124. Elle en tire la conclusion que Solon voit dans son action de libérateur et de législateur « deux formes équivalentes de l'agir, aussi essentielles l'une que l'autre ».

10 SOLON, fr. 30, 17, 20 G.-P. 
l'espace politique un talent poétique ${ }^{11}$. C'est, en effet, parce qu'il se perçoit comme poète, c'est-à-dire comme détenteur d'une alètbeia, dont M. Detienne ${ }^{12}$ a montré que, dans la Grèce archaïque, elle était non-oubli, mémoire - l'alpha étant alors perçu comme privatif - tout autant que vérité, que Solon s'estime habilité à placer sa parole au centre d'Athènes. Cette prétention, il l'affiche au temps de Pisistrate encore, lorsqu'il tente, au nom de cette même alètheia, de s'opposer au régime naissant du tyran. Et, comme ses concitoyens le traitent alors de fou, parce qu'il use d'arguments devenus pour eux surannés, il leur répond imperturbablement ${ }^{13}$ :

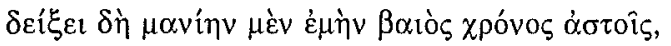

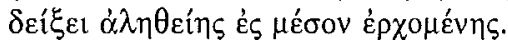

Un temps court montrera ma folie aux citoyens, il la montrera lorsque la vérité viendra au centre.

C'est cette vérité qui est Mémoire et don des Muses que Solon inlassablement entend réaliser au nom de la justice et que, par sa parole, il affirme mettre au centre de la cité, quand il en rédige les lois. La loi, dans cette conception, est la parole de Solon devenue écrit et qui, par là, s'impose, même en dehors de la présence physique du législateur. L'écriture de la loi - sa promulgation - permet ainsi à Solon de réunir en sa personne ce roi de justice de jadis qui rendait des sentences justes et ce poète que la Théogonie évoque tout aussitôt après lui, parce qu'il est détenteur d'une même parole inspirée ${ }^{14}$ :

Celui qu'honorent les filles du grand Zeus lorsqu'à sa naissance, elles le regardent parmi les rois nourrissons de Zeus, et qu'elles versent sur sa langue une douce rosée, celui-là de sa bouche répand des paroles douces. Tous les gens ont le regard fixé sur lui lorsqu'il rend ses jugements avec de justes sentences. Il parle de façon sûre dans l'agora, et il le fait promptement, sachant comment faire cesser de grandes querelles. Car c'est à cela que l'on reconnâtt les rois sages, à ce

11 G. NAGY, Theognis et Mégare, in RHR, 201 (1984), p. 246, suggère qu'une même adéquation entre beauté musicale et intégration politique et sociale, est présente chez THÉOGNIS, 15-18, où le mariage de Cadmos, auquel participent Muses et Charites, renvoie à Harmonie, son épouse, même si elle n'y est point nommée, en une harmonie qui est tout autant musicale que sociale.

12 Par une étude du champ sémantique du mot alètbeia, M. DETIENNE, La notion mytbique d'ALETHELA, in REG, 73 (1960), p. 27-35, a montré que l'alpha initial y est perçu comme privatif à l'époque archaïque. Dès lors la mémoire du poète, du devin, du roi de justice, qui se réclament d'une même parole inspirée, s'affirme vérité en même temps que non-oubli, thème que développe M. DETIENNE, dans Les Maîtres de Vérité, Paris, $1981^{2}$, en insistant sur l'ambivalence de la parole (ibid., p. 51-80) qui, si elle peut être véridique, peut aussi être trompeuse [cf. HÉs., Th., 26-29 (supra, n. 1) et SOLON, fr. 25 G.-P. : Les poètes mentent beaucoup].

13 SOLON, fr. 14 G.-P. = D. L., I (Sol.) 49.

14 HÉs., Th., 81-103. Sur ce passage et la sacralisation de la mémoire dans une société orale : J.-P. VERNANT, Aspects mytbiques de la mémotre et du temps, in Mytbe et pensée chez les Grecs, Paris, $1982^{2}$, p. $80-89$. 
qu'aux hommes qui ont souffert un dommage, ils savent dans l'agora facilement donner satisfaction, en réprimandant avec des paroles douces. Et quand il s'avance à travers l'assemblée, on l'honore comme un dieu, pour sa douce retenue, et il se distingue parmi ceux qui sont réunis. Tel est le don sacré que les Muses font aux hommes. Car c'est par les Muses et l'archer Apollon qu'il y a sur terre des aèdes et des citharistes, comme par Zeus, il est des rois. Heureux celui que les Muses chérissent. De sa bouche coulent de douces paroles. Et si quelqu'un éprouve du chagrin dans son cœur, souffre d'une douleur fraîche et se dessèche dans la tristesse, que vienne un chanteur, serviteur des Muses, qui célèbre les hommes d'autrefois et les dieux bienheureux qui habitent l'Olympe,

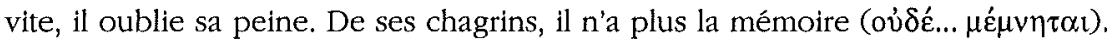
Vite, le présent des Muses l'en a détourné.

Chez Hésiode, la parole du poète qui est Mémoire détourne du temps présent dont elle fait perdre la mémoire, pour ouvrir sur le temps mythique qui, par son évocation, console du premier. Cette parole poétique qui, par le mythe, dissout la triste écume des jours ne débouche cependant pas chez lui sur un changement politique. Hésiode peut morigéner les rois, comme il peut morigéner son frère, il peut aussi prier Zeus de réaliser la justice en des accents que retrouve Solon pour célébrer l'Eunomia ${ }^{15}$; il n'est pas l'agent personnel du changement, quand bien même l'appelle-t-il implicitement de ses voux en évoquant l'ordre civique idéal. Cet ordre, par contre, Solon prétend le réaliser dans cette cité perturbée qu'est Athènes, au début du VIe siècle, grâce à son pouvoir poétique qui lui permet d'y placer l'alètheia au centre. C'est, en effet, parce qu'il se définit comme poète, détenteur d'une parole inspirée que, dès le début de sa carrière politique, à cette époque où Salamine est encore aux mains des Mégariens, il utilise le poème comme argument politique, de façon à inciter ses concitoyens à prendre l'île aux Mégariens. Il se dit alors béraut de lui-même, messager mandaté par lui-même, au nom du pouvoir poétique qu'il détient $^{16}$ :

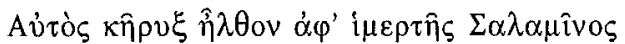

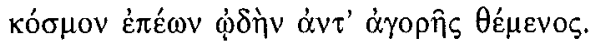

15 W. JAEGER, Solons Eunomie, in SDAW, 11 (1926), p. 69-85 = Five Essays, Montréal, 1966, p. 77-99, fait justement remarquer ( 1 bid., p. 96-97) que SOLON, fr. 3, 32-39 G.-P. comporte les mêmes accents religieux que HÉS., $T r$., 1-10, avec une différence dans le style cependant : là où Hésiode utilise le parallélisme, Solon emploie le chiasme.

16 SOLON, fr, 2 G.-P. = PLUT,, Sol, 8, 2, qui précise que cette élégie avait pour titre Salamine et qu'elle comportait cent vers d'une grande beauté. - Sur le rôle de représentant du pouvoir que détient le héraut et qui est nécessairement important dans une société orale : Louise-Marie WÉRY, Le fonctionnement de la diplomatie bomérique, in RIDA, 14 (1967), p. 169-205 = Die Arbeitsweise der Diplomatie in Homerischer Zeit, in E. OLSHAUSEN et Hildegard BILLER (éds), Antike Diplomatie, Darmstadt, 1979, p. 13-53; EAD., Le Meurtre des bérauts de Darius en 491, in AC, 35 (1966), p. 468-486. 
Héraut de moi-même, depuis l'aimable Salamine, je suis venu, pour chanter l'ordre des mots en un poème ${ }^{17}$, en lieu et place d'un discours dans l'agora.

Cet ordre qui est celui du vers, comme il est par analogie celui de la cité, il ne le conçoit cependant pas comme arbitraire, mais comme résultant de cette mesure qui préside à l'ordonnancement du vers, comme elle préside à l'agencement civique. Le poète, dit-il, dans une définition qu'il donne de différents métiers, tous itinérants, est détenteur de la mesure qui fonde sa sopbia ${ }^{18}$ :

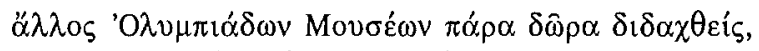

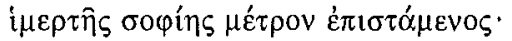

Un autre, instruit du don des Muses de l'Olympe, connaît le mètre de son art aimable.

Cet art, cette sopbia, qui est tout autant expertise politique que poétique, même si c'est, aux yeux de Solon, la sopbia du poète qui fonde celle du politique, il l'estime cependant difficile à atteindre. Car elle est fondée, dit-il, sur la mesure qui détermine les limites de toute choses $^{19}$ :

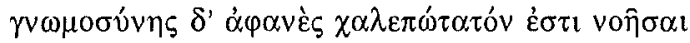

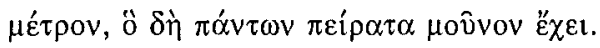

Il est très difficile de percevoir la mesure invisible de la sagesse qui seule fixe les limites de toute chose.

Comprendre la mesure, c'est percevoir la limite, se la fixer pour terme. Ainsi, de même que le mètre repose sur une alternance de temps forts et de temps faibles, la cité de l'Eunomia repose sur une harmonie qui concilie les opposés. C'est cette harmonie que Solon prétend atteindre en instaurant à Athènes un ordre politique analogue à celui de la poésie et qui, à ce titre, repose sur la mesure ${ }^{20}$.

Cette perception que Solon se fait de son activité politique comme découlant de sa fonction poétique a cependant été fort peu mise en évidence par ses

17 Même type d'apposition en SOLON, fr. 3, 17 G.-P. : M.I. LINFORTH, Solon the Atbenian, Berkeley, 1919, p. 201.

18 SOLON, fr. 1, 51-52 G.-P. = STOBÉE, III, 9, 23.

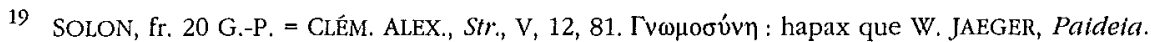
La formation de l'bomme grec, Paris, 1933, tr. fr., éd. Gallimard, 1988, p. 186, traduit par « discernement», en soulignant la présence dans ces vers de l'idée de mesure et de limite, fondamentale dans l'éthique grecque.

20 Harmonie et mesure sont de même associées en SOLON, fr. 5 G.-P. = A.P., V, 3 : aux chefs du peuple qui manquent de mesure, Solon annonce que le peuple n'obéira pas et que cela ne sera pas harmonieux (artia) pour eux. La même idée est reprise en SOLON, fr. 8 G.-P. = A.P., XII, 2. 
commentateurs antiques comme par les historiens contemporains qui pourtant inlassablement se sont penchés sur son ouvre ${ }^{21}$. Ceci pour deux raisons, semble-t-il. Cette vision intériorisée que Solon se fait de son ceuvre politique ne fut pas celle de ses concitoyens qui le virent avec un autre regard, non pas tant comme le poète, réalisateur de l'Eunomia, que comme le tyran potentiel qui réaliserait la cité juste, selon la manière dont eux l'appréhendaient, et qui n'était pas celle d'une loi égalisatrice, mais d'une répartition égalitaire de la terre civique. De Solon, le dèmos - les classes populaires - attendait qu'il réalise le partage égal de la terre civique, son isomoiria, et qu'il s'empare de la tyrannie, nécessaire pour atteindre cet objectif. Car le partage égal de la terre civique devait nécessairement rencontrer l'opposition des gros propriétaires. Or Solon refuse et le principe de l'isomoiria et celui de la tyrannie, nécessaire pour le réaliser, défendant ainsi sa position face au dèmos ${ }^{22}$ :

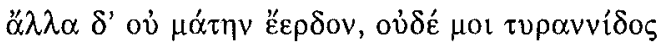

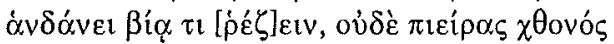

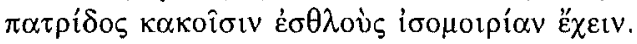

Mais je n'ai pas agi à la légère. Ma politique exclut de faire quoi que ce soit avec la violence de la tyrannie et que, de la terre grasse de la patrie, les nobles aient une part égale aux gens de rien.

Ainsi reste-t-il cet aristocrate, défenseur des intérêts aristocratiques qu'il estime fondés car, selon lui, il est juste que les aristocrates possèdent plus de terres que les kakoi. L'isomoiria de la terre ne peut donc être à ses yeux le moyen de réaliser la cité des bomoioi à laquelle, comme le dèmos cependant, il aspire $^{23}$. Cette cité, c'est par la promulgation de la loi qui rend chaque Athénien égal devant elle, et non par le partage égal de la terre civique, qu'il entend l'établir. Aristocrate et poète, défenseur d'Athènes certes, mais aussi d'une conception de la cité qui reste aristocratique, Solon parle ainsi au dèmos

21 I.M. LINFORTH, op. cit. (n. 17), p. 179, constatant que le mot $\alpha \rho \tau \imath \alpha$ est un des mots favoris de Solon, n'en tire cependant aucune conclusion sur la manière dont il conçoit son activité politique qu'il situe, quant à lui, dans un contexte analogue à celui de la lutte entre la bourgeoisie et le prolétariat à la fin du XIXe siècle : il s'agit pour la classe dirigeante de se faire de l'argent (money making, ibid., p. 203). C'est en fonction de la crise des années 30 que W.J. WOODHOUSE, Solon the Liberator. A Study of the Agrarian Problem in Attika in the Seventh Century, Oxford, $1938=$ New York, $1965^{2}$, étudie à son tour la libération de la terre, opérée par Solon, qu'il dessine comme un personnage messianique, notamment dans son introduction. Dès lors l'attention se trouve détournée des aspects archaïques du personnage qui ne sont exploités ni par A. MASARACCHIA, Solone, Florence, 1958, ni même par M. DETIENNE, op. cit. (n. 12), p. 34, n. 28, qui cite SOLON, fr. 3, 15 G.-P., pour souligner que Dikè est chez lui celle qui sait ce qui est et ce qui a été, mais non SOLON, fr. 14 G.-P. où Solon se présente comme maître de vérité.

22 SOLON, fr. $29^{\mathrm{b}}$ G.-P. - Sur le contexte historique spécifique dans lequel furent prononcés ces tétramètres : H. VAN EFFENTERRE, ait. cit. (n. 5), p. 114.

23 Le terme ópó́w̧ est capital dans SOLON, fr. 30,18 G.-P., comme on l'a montré notamment dans Louise-Marie L'HOMME-WÉRY, Solon, libérateur d'Eleusis dans les "Histolres" d'Hérodote, in $R E G, 107(1994)$, p. 376-379. 
un langage qui, pour lui, sans doute est clair, puisqu'il se défendra d'avoir réalisé autre chose que ce qu'il avait promis ${ }^{24}$, mais qui néanmoins ne le fut pas pour les kakoi d'Athènes, persuadés qu'il saisirait la tyrannie et qui ne furent pas convaincus par les motifs de son refus ${ }^{25}$ :

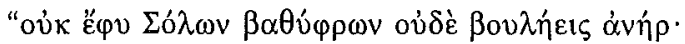

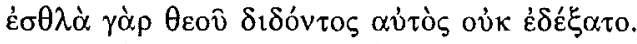

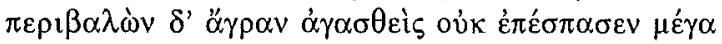

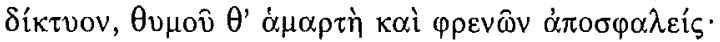

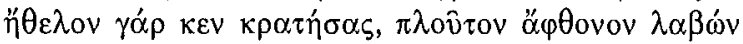

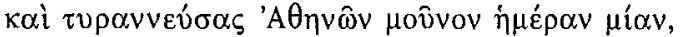

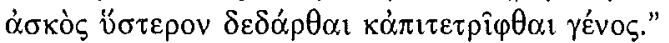

«Solon n'est pas un homme avisé, ni malin, puisqu'il a refusé les beaux présents qu'un dieu lui donnait. Alors qu'en homme admiré, il avait enveloppé sa proie, il n'a pas su tirer le grand filet. Le courage et l'intelligence lui ont fait défaut en même temps. »

Effectivement. Si je l'avais voulu ${ }^{26}$, comme prix du pouvoir, de l'acquisition d'une abondante richesse et de l'exercice de la tyrannie - durant un seul jour, à Athènes -, j'aurais accepté d'être ensuite écorché vif et de voir ma famille anéantie.

Le fait néanmoins qu'il y eut débat suppose qu'il y eut ambiguité et que ce n'est pas d'abord et principalement comme poète détenteur de l'alètheia que Solon fut perçu, à l'époque où, après avoir libéré Eleusis, il rédigea les lois d'Athènes, mais bien comme un tyran en puissance. En effet, par le fait même qu'il avait libéré Athènes, en s'appuyant sur une force militaire pour le faire, puis qu'il avait revendiqué l'autorité pour en promulguer seul les lois, c'est la personnalité du tyran que, sans le vouloir, il rejoignait ${ }^{27}$. Et ceci quand bien même s'en défend-il dans ses poèmes, y soulignant ce qui le sépare de cet homme mal intentionné et avide qu'est le $\operatorname{tyran}^{28}$ :

24 SOLON, fr. 30, 1-2 G. -P.; fr. 29b, 6 G.-P.

25 SOLON, fr. $29^{\text {a }}$ G.-P. = PLUT., Sol, 14, 9.

26 Les trois derniers vers de SOLON, fr. $29^{a}$ G.-P. sont une réponse de Solon à ses adversaires. D'où le passage assez déroutant de la troisième personne à la première. Le yò̀ $\rho$ est ironique. Solon semble d'abord donner raison à ses détracteurs, avant de les contrer. - Sur la variété des tons chez Solon qui vont de la dignité solennelle dans les élégies à l'humour sarcastique dans les tétramètres : P.-E. EASTERLING et B.M. KNOX (éds), The Cambridge History of Classical Literature, I, 1985, p. 152.

27 J. F. MCGLEW, Tyranny and Political Cilture In Ancient Greece, Ithaca - Londres, 1993, p. $52-$ 83, montre que la tyran archaïque prend la succession du roi de justice que dépeint HÉs., Th., 80-96, en renversant les aristocraties corrompues, les monarcboi en place, et en assurant par de nouvelles institutions, ainsi les juges itinérants nommés par Pisistrate (A.P., XVI, 5), la fonction de justice, tandis qu'il souligne (ibid., p. 94) que la fonction d'arbitre, de diallaktès, que Solon détient en $594 / 3(A . P ., \mathrm{V}, 1)$, rejoint celle du tyran.

28 SOLON, fr. 30, 20-27 G.-P. 


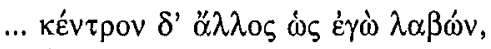

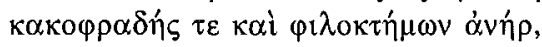

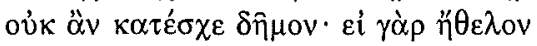

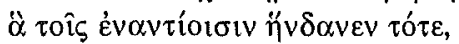

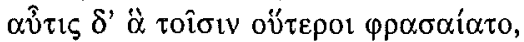

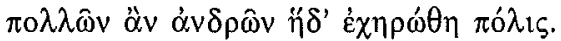

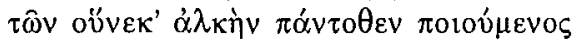

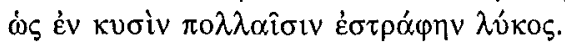

Si un autre, comme moi, avait pris l'aiguillon, un homme mal intentionné et avide, il n'aurait pu retenir le peuple. Car, si j'avais voulu ce que souhaitaient alors mes adversaires ou inversement ce que l'autre parti avait en tête à leur égard, c'est de beaucoup d'hommes que la cité eût été privée. C'est pour cela que, ramassant de partout ma force, j'ai fait front, comme au milieu de chiennes nombreuses, un loup.

Pourtant, alors même qu'il entend se distinguer de ce tyran qui, par intérêt et goût du pouvoir, aurait menacé Athènes, la conduisant à la guerre civile et la vidant de ses hommes, c'est encore la personnalité de ce tyran que Solon rejoint quand il se présente comme tenant seul l'aiguillon, et c'est l'image de ce tyran devenu vulnérable qu'il évoque encore quand il se voit loup solitaire, environné par les chiennes de la citée ${ }^{29}$.

Personnage ambigu, à mi-chemin d'une Athènes aristocratique et de cette Athènes démocratique qui n'existe pas encore, Solon, poète, devenu homme politique pour y réaliser une Eunomia qui se veut conciliation des contraires et qui, à ce titre, n'entend pas rompre avec la cité aristocratique, mais la tempérer dans ses excès, ne fut donc pas clairement perçu comme tel par ses contemporains. Il ne le fut pas davantage par l'Athènes du $\mathrm{IV}^{\mathrm{e}}$ siècle qui réinterpréta son ouvre à la faveur des conflits politiques qu'elle-même connaissait et vit en lui un $\tau$ ô $\delta$ ń l'histoire d'Athènes, face à l'avidité et aux intérêts de la classe dominante des plousioi, qui sont davantage ceux du IVe siècle que ceux de l'époque de Solon $^{30}$. Or cette vision que l'Athènes du IVe siècle se fait de Solon, parce qu'elle se trouve immortalisée dans l'interprétation que la Constitution d'Athènes donne de son ouvre politique, sur base des poèmes qu'elle cite, passe dans la recherche contemporaine qui, elle aussi, voit en lui un démagogue qui s'exprime par la voie de la poésie, et non un poète qui, à ce titre, prétend réaliser Athènes.

Enfin, par le fait même qu'il place ses lois au centre d'Athènes, comme autant de manifestations de cette alètheia dont il se veut le détenteur, Solon

29 Sur le loup-tyran à l'époque archaïque: M. DETIENNE - J. SVENBRO, Les loups au festin ou la cité impossible, in QS, 9 (1979), p. 3-23 = La cuisine du sacrifice en pays grec, Paris, 1979, p. 215237.

$30 \quad$ A.P., II, 2. 
leur enlève ce caractère sacré dont elles étaient parées jusque-là. Déposées au centre, inscrites sur ces kurbeis où elles se veulent visibles par tous, lisibles pour tous ${ }^{31}$, ses lois y sont sujet d'observation et de critique, de même qu'objet possible de révision, de modification, auxquelles peuvent s'ajouter des promulgations nouvelles ${ }^{32}$. Dans l'agora, elles deviennent le bien d'Athènes, cessant par là-même d'être le privilège d'un roi de justice dont le règne s'achève, alors même qu'il se réalise. Conscient sans doute de cette perte de prestige que subissaient ses lois par le fait même de leur promulgation, Solon en rédigea une seconde version en vers, en même temps qu'il demandait à Zeus de leur accorder bonne chance et gloire ${ }^{33}$ :

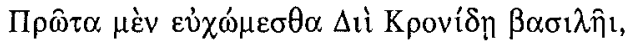

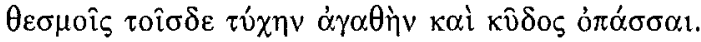

Et tout d'abord prions Zeus, fils de Kronos, Roi, d'octroyer à ces lois bonne chance et gloire.

Ainsi, parce qu'il utilise l'écriture pour rendre effective l'alètheia dont il dispose, Solon met nécessairement fin au pouvoir que lui donnait une parole sacrée. La cité de l'Eunomia qu'il voit comme la réalisation d'un ordre poétique, fondé sur une harmonie qui est conciliation des opposés, est destinée à s'effacer devant celle de l'isonomia où le pouvoir est placé dans les mains de chaque citoyen, et non plus dans celles d'un maittre de vérité solitaire. Tel Solon, dont la personnalité frôla celle de ce tyran qu'il se refusa à devenir, mais non sans devoir pour cela quitter Athènes.

Louise-Marie L'HOMME-WÉRY

$22 \mathrm{~A}$, rue de Roloux

B - 4347 FEXHE-LE-HAUT-CLOCHER

31 Sur ce mode d'exposition, l'étude de E. RuschENBUSCH, Solônos Nomoi: Die Fragmente des Soloniscben Gesetzeswerkes mit einer Text- und Überlieferungsgeschicbte, in Historia, Einz., 9 $(1966)=(1983)^{2}$, p. 23-25, reste déterminante.

32 HDT., I, 29, signale que les Athéniens avaient juré de ne pas modifier pendant 10 ans les lois que Solon leur donnerait. Mais cela n'empêchait pas les modifications ultérieures, $A . P$., VII, 2 , donne le terme de 100 ans qui est improbable.

33 SOLON, fr, 40 G.-P. - PLUT., Sol., 3, 5, qui cite ce fragment de Solon, affirme qu'il ouvrait la version en vers que Solon avait entreprise de ses lois. Il faisait ainsi rentrer Dikè dans un dire poétique : LORAUX, art. cit. (n. 9), p. 123-124. 